\title{
Prediction of kinase inhibitors cross-reaction on the basis of kinase ATP cavity similarities: a study using PKSIM protein similarity score A Zaliani*, C Mueller and M Rarey
}

Address: Bioinformatik Zentrum Uni-Hamburg, Bundesstrasse 43, 20146 Hamburg, Germany

* Corresponding author

from 3rd German Conference on Chemoinformatics

Goslar, Germany. II-I3 November 2007

Published: 26 March 2008

Chemistry Central Journal 2008, 2(SuppI I):PI9 doi:I0.II86/I752-I53X-2-SI-PI9

This abstract is available from: http://www.journal.chemistrycentral.com/content/2/SI/PI 9

(C) 2008 Zaliani et al.

In latest years, there has been an increasing attention to chemoinformatic and modeling methods enabling selectivity predictions for small molecules in case of same mechanism of action. Selectivity profiling of small molecules is emerging as one of the most expensive, though yet unmissable, task in drug discovery and cross-reactivity prediction as one of the most challenging chemoinformatic field, accordingly.

Following the widely accepted similarity principle [1] for which structurally similar compounds should act similarly, we have to expect also that similar proteins cavities recognize similar compounds. For historical reasons, kinase inhibition is one of the most attractive field to test new predictive methodologies as ATP antagonism is probably the most inherently problematic mechanism of action from a cross-reactivity standpoint.

In the present work, we thus focus on kinases, approaching the problem with the use of PKSIM, a novel software tool for protein active site comparison. PKSIM is based on finding interaction spots developed for docking calculations [2] and the maximal overlap thereof. The pairwise PKSIM spots score has been used to rationalize a published cross-reactivity panel of a series of Kinase inhibitors [3]. We also performed a new classification of 584 kinases on the basis of the similarity score which allowed us to build a "classification tree" of the currently known kinases ATP cavities. This tree, as expected, showed marked differences from the known kinome tree based on sequence alignment [4]. Moreover, from this training set panel a simple empirical rule has been found to predict the extent of selectivity for a general ligand active on a given kinase ATP binding site.

Extending the similarity concept further to the family of crystallized protein targets, we can now apply and optimize the present tool to different and important tasks: 1) to check easily if, and at which extent, old drugs with their selectivity profiles, are worth to be tested on new target proteins according to their target similarities; 2 ) to predict risks of possible off-target toxic effects on the basis of unprecedented cavity similarities of targets and finally, 3) through the similarity assessment of every newly crystallized cavity, try to infer its recognition potential towards known chemical space.

\section{References}

I. Kubinyi H: Perspectives in Drug Discovery and Design. 1998, 9-I I (0):225-252

2. Schellhammer I, Rarey M: Proteins 2004, 57(3):504-5I7.

3. Fabian MA, et al.: Nature Biotechnology 2005, 23(3):329-336.

4. Manning G, et al:: Science 2002, 298:19|2-1934. 